\title{
Background and the current healthcare ranking systems in the US
}

\author{
Cyrus Engineer* \\ Clinical Professor and Director, Health Care Management, Department of Interprofessional Health Studies, Towson University, Maryland, USA
}

For many individuals facing serious medical problems in need of healthcare, a decision tool concerning the best health facility to visit is very pivotal. For the United States, this was lacking until 1990 when hospitals were rated, rather than ranked by the US News and the World Report. The rating was done on the basis of 12 major specialties. It was until 1991 when hospitals were actually ranked. In 2015 and 2016, best hospital ranking documented according to the American Hospital Association Annual Survey of Hospital findings was done. The ranking was done taking into consideration different specialties in order to help clients identify with the best healthcare facilities for delicate and complicated medical procedures such as placement of heart stents in a geriatric with multiple comorbidities [1]. The methodology employed in the Best Hospital rankings was set by the National Opinion Research Center in the early 1990s [2]. Since the establishment of this methodology, it has been fine-tuned over time to encompass vital factors such as patient safety, as at 2009. The specialties considered in the rankings too have been adjusted over time. For instance, HIV care which once featured among the specialties has been scraped off considering that the management of these patients is currently more outpatient based rather than inpatient.

The specialties considered within the methodology include: urology, rheumatology, Psychiatry, Rehabilitation, pulmonology, orthopedics, ophthalmology, neurology \& neurosurgery, nephrology cancer, cardiology \& heart surgery, diabetes \& endocrinology, ear nose \& throat, gastroenterology \& GI surgery, geriatrics and gynecology [1]. The Index of Hospital Quality took into consideration twelve out of the sixteen specialties; that is, excluding ophthalmology, psychiatry, rehabilitation and rheumatology. Performance rating then was done considering hospital structure, processes and outcomes. Structure addressed availability of adequate and quality staff directed towards patient there. These include sufficient nursing staff as well as other medical staff as well availability of up to standard medical equipment's alongside other technologies requisite in ensuring quality patient care. Process addresses establishment of diagnosis, methods of administering treatment, prevention and patient education on various issues. Evaluation of process is important in determining the sustainability of a health facility in provision of standard medical care. Outcomes include death, complications resulting from treatment especially preventable ones, harm to patients and preventable readmissions among others.

Patient safety is also among the methodologies used in hospital ranking. This includes all risks that patients are exposed to but do not succumb. Initially, when data driven methodology was employed, structure, process and outcomes were equally weighted as far as their effectiveness in ranking were concerned. With the introduction of the patient safety element in 2009 , however, its weight was equally divided amongst outcomes and processes. With the rising concerns as pertains to patient safety, this criterion was given more weight in 2014 to 2015 . The table below shows the various components used in the ranking of health systems, as well as their individual weight contributions to the overall score [Table 1].

According to Rothberg, et al. [3] however, this system which entails public reporting tends to misguide the populations or confuse them further. This is because some of the reports given by the hospitals during public reporting is a little exaggerated and may not reflect the true state of the patients. Besides, much as the reporting may be done to have patients select the best healthcare provider, it has been noted that a good number of patients may not pay keen attention especially to elements such as mortality reports. Such would therefore still rely upon third party experience and referral by friends and relatives in selection of their healthcare service providers [4]. A prominent setback of the hospital ranking systems in the US is that the different ranking systems rarely agree. It is in many cases that a hospital that ranked highly when ranked using one system did not even feature among the top when rated using another system. This has thus brought so much confusion because patients are then left wondering which system is more credible [5].

\section{Healthcare Ranking Systems in South East Asia and Middle Eastern Countries}

In Middle Eastern countries, hospital ranking is done taking into consideration six major criteria. These include: The extent to which a health facility applies international quality standards, efficiency and proficiency of the medical personnel, the level, number and quality of scientific researches, success rate of medical and surgical procedures, the range and quality of services provided, low incidence of medical errors as well as treatment failures and the level of training opportunities for personnel. In order to improve the performance of

Table 1. Overall Weight by Component.

\begin{tabular}{|c|c|}
\hline $\begin{array}{c}\text { A } \\
\text { Component }\end{array}$ & Weight (\%) \\
\hline Outcomes & 32.5 \\
\hline Structure & 30.0 \\
\hline Process & 27.5 \\
\hline Patient Safety & 10.0 \\
\hline
\end{tabular}

Correspondence to: Cyrus Engineer, Clinical Professor and Director, Health Care Management, Department of Interprofessional Health Studies, Towson University, Maryland, USA, Tel: 410-704-6280; Fax: 410-704- 4965; E-mail: cengineer@towson.edu

Received: November 22, 2017; Accepted: December 23, 2017; Published: December 26, 2017 
health facilities here, health systems stakeholders are incorporating a management model known as Population Health Management (PHM). This aims at providing the highest quality of services to the patients. Under this model are three major elements: focusing on the health outcome of the whole population, coordinating the various sects of healthcare provision which include curative, palliative, preventive and health promotion. Lastly, is seeking to involve patients in making decisions as far as settling their healthcare needs is concerned [6]. This model alongside the ranking system is bound to see the hospitals in Middle East grow to a higher level and effectively compete globally.

Southeast Asia has got diverse populations and thus a diverse nature of health systems. These health systems are at different stages of evolution and thus great socioeconomic development [7]. This is among the factors that have made hospital ranking a chief necessity, if these hospitals are to develop almost equitably while providing the highest standards of care. Healthcare ranking in South East Asia is done with respect to the World Hospitals Ranking on the Web, whose basis is on various processes of generating and communicating scientific knowledge. The aim of these rankings is to motivate people globally to post quality scientific material on the web for purposes of access by colleagues and other people. Through these postings institutions can be rated on the basis of scientific activities, their performance in these activities as well as the impact the information has upon its readers. The key objective of this ranking is to encourage web publication by various hospitals, making an evaluation of how committed these organizations are to electronic distribution; and to fight the academic divide existing even amongst hospitals in developed countries. In the web ranking system, hospitals are rated judging from their scientific output, workshop write-ups, seminars, multimedia among other elements. In the event the web ranking of an institution falls below their expected standard, a review of their web policy is advocated for with a goal of increasing the volume and quality of their electronic publications.

The ranking system in Asia has resulted in improved health facilities thus worldwide prominence in various fields [7]. For instance, hospitals in Thailand are now well known worldwide for their refined art in cosmetic surgery. Malaysia on the other hand is well known in administration of the best forms of fertility treatments. Honk Kong has established its niche of expertise in cardiology and other related treatments. Though there exist many healthcare institutions in the United States which were constructed way before those in Asia, those in Asia have been equipped with the best and most recent equipment, resulting in more accurate diagnoses as well as less invasive surgical procedures. In a recent report by the World Health Organization (WHO), some hospitals in Hong Kong and Singapore are considered best in the world. With the increasing demand for better methods of treatment year in year out, Asian countries are putting in place measures to ensure they keep up the pace. Thus, the future of Healthcare in South East Asia is promising.

\section{Healthcare System in India}

India is among the developing countries in the world. It has got a large population, most of whom have no health insurance covers. Thus, most people have to spend cash from their pockets when seeking after health services [8]. Some health care facilities here charge medical fees which would be best considered as being exorbitant yet they lack the necessary clinically proven protocols of administering treatment. Judging by international standards, the levels of health insurance in India are very low. The available private insurance schemes available do not cover for consultation fees or medical fees and therefore do not effectively meet the population health insurance needs. The private medical sector serves approximately 70 percent of people in India. This is attributed to the fact that the quality of service provided in the public sector is extremely poor leaving people with a more expensive option, the private sector [9]. The public sector majorly caters for the rural population, which is a factor that has contributed to the low standards in these public hospitals because health professionals are a little reluctant to visit rural areas due to factors such as insecurity. As such, these public facilities are manned by inexperienced and unmotivated interns who have to be there for the sake of meeting their curricular requirements.

According to Britnell [8] the patients in India also lack information about various hospitals, their doctors, qualifications, competencies as well as a record of their past achievements. As a result, choices of a medical facility are made blindly following referrals by friends and relatives as well as marketing appeal created by marketing teams of various hospitals, who definitely over-rate their facilities. Others make their choices depending on third party experiences or proximity to a health facility with minimal knowledge of the standards of care provided therein [10].

\section{Hospital Ranking Model for India}

Considering the state of Health Care systems in India, there needs to be a well laid down mechanism of hospital ranking. This needs to be done factoring in the various stakeholders in the health sector. These include yet not limited to hospital administrative bodies, doctors, nurses, paramedical staff, health insurance companies as well as the most important of them all; the patient. This ranking system is vital because it will enable all the stakeholders to make informed and unbiased choices and opinions as far as health facilities are concerned based purely on the quality of health care provided by various facilities. Ranking will also enable the managerial bodies of the respective health facilities to work towards attaining the highest standards of health care service provision in a bid to match up with their counterparts in the industry. The proposed model will enable patients to select facilities that guarantee the highest standards of care, medical staff will be able to identify with the right health care institutions to work with, quality highly ranked hospitals will benefit from a larger client base, insurance firms can use the rankings to select the institutions in which their patients can be covered, whereas the non-Governmental Organizations can also make an informed choice of which health institutions to make their donations to.

Being a developing country, much simpler criteria of ranking hospitals will be proposed. This takes into consideration resources, efficiency, patient-centeredness, safety, equity, clinical excellence and timeliness. Availability of resources is measured to determine financial flexibility and strength. Efficiency will be determined considering factors such as the turnaround time per patient depending on various procedures, for instance caesarean sections. It can also be monitored depending on utilization of human resources as well as medical supplies. On safety, the hospital environment should be safe to all patients and health workers. For instance, sterile gloves should be used for surgeries to minimize chances of nosocomial infections. Protective gears should also be available for the medical stuff to minimize chances of contracting infections and diseases from patients or their working environment.

Patient preferences should be key in guiding clinical decision; equity should be maintained through and through regardless of race, ethnicity or gender among other personal characteristics. Clinical excellence must be guaranteed through provision of evidence-based medicine, whereas 
timeliness should never be compromised by ensuring patients receive medical attention soonest possible. The assessment methodology that will be used will be based on the following factors:

\section{Access \& continuity of care}

This aspect will analyze the location of the health facility in relation to the patient base it serves, ease of accessibility which encompasses availability of ambulance services for patients in critical condition, as well as the number of hours the health facility operates per day.

\section{Patient care}

This considers whether there exist standard protocols governing patient care and whether those protocols are followed. Regular checks should be done to ensure minimal breech in this element.

\section{Consistent quality improvement}

This encompasses provision of continuous trainings for medical personnel to ensure they keep up with the latest trends in the medical field. It also entails monitoring of errors and continually putting in place measures to ensure they are on the minimum. Monitoring of deaths and identifying preventable deaths is a vital element of this too.

\section{Infection control}

This considers the equipment used by the hospital, whether those that need sterilization are well sterilized, where procedures such as vaginal exams are done using sterile gloves, whether the surgical equipment and drapes used in theatres are sterile as well as the existing channels of waste disposal. It also considers the incidence of hospital-acquired infections as well as the mechanisms put in place to deal with them.

\section{Human resource management}

This considers recruitment and training of medical personnel. It also involves confirmation of accreditation of medical personnel before employment.

Having considered the above factors and accordingly ranked hospitals, the hospitals are informed of their ranking. Any disgruntled party is allowed to appeal. The final results are then posted in a forum accessible to the patients and all other stakeholders. The findings, however, are subject to reviews for instance when a hospital acquires new material inputs, or when one is noted to be a little redundant in comparison to the others within the industry.

\section{Challenges and Opportunities in Implementing the Ranking Model in India \\ Opportunities}

As earlier alluded to, India is Continent in which majority of its patients lack insurance covers; and even those with insurance covers, especially the private insurance covers, lack complete cover for their health and still have to foot a few expenses such as consultation fees from their pockets. As such, majority of these people would be more grateful and feel less financial loss if value for their money would be guaranteed. As such, these people would greatly welcome and support the hospital ranking system. Obtaining information such as timeliness from patients therefore would be done with great ease since cooperation will be at its peak.

Hospital ranking will also be an avenue of lowering the cost of obtaining health care. This will happen through patients making more informed choices of the best health care facilities therefore resulting in market forces which will in the long run lower prices and improve quality as hospitals compete to raise their ratings, as well as attract a larger client base. Though patients may not quite use the ratings to select their service providers, it has been identified from other countries that public release of performance and rating of health facilities improves their quality of service provision since no service provider delights in low ratings, especially when made known to the entire public. This factor will therefore lead to tremendous improvement in quality of services provided including in the rural based hospitals in India, whose current state appalling.

The ranking system has worked well in most of the developed countries. Most of the developing countries are yet to embrace this system, which could explain why health provision in these countries is still in a sorry state. Given the positive impacts the ranking system has had upon developed countries, it could serve as an opportunity in this quest to have it implemented in India, as it will receive massive support from the various stakeholders. Though new, it is a system that has been tested in other nations and transformed health care from one level to another. This motivation thus guarantees immense cooperation from the citizens, governments, health facility owners as well as non-governmental institutions; with minimal resistance if any. This system too is appropriate for a continent such as India which has got virtually all the health specialists and considerably good technology, which explains why it has for a long time served as a referral center for complicated medical conditions especially from the African continent. Besides, having it enforced here benefits not only India but other continents as well which rely on India for complicated medical consultations.

\section{Challenges}

This being an absolutely new system, it is bound to face its challenges. Whereas its implementation is aimed at having patients make informed choices concerning health provision, it may end up resulting in confusion. This is because much as the patients may follow through the rankings to select their health providers, majority would still want to select their health providers based on other patient's experiences. This would therefore affect the end objective of improved healthcare facilities, since once a facility still retains its customer base despite its low ranking, there would be no propelling force to keep up with other hospitals within the industry.

Some of the criteria used in ranking such as mortality rates may not be of great benefit since despite the information, clients may still go to institutions with the highest mortality rate, especially those who have been managed over a long period of time over chronic illnesses and are they themselves satisfied with the outcomes. Some of the criteria may also result in confusion among patients. For instance, a patient suffering from angina may be confused between whether to access a hospital that is closest considering the nature of the condition which in some cases is a medical emergency, or one that is distant but possesses the best cardiologist or equipment. In the event these two aspects do not exist within the same hospitals; patients may end up confused and end up making more unwise decisions.

Besides the above challenges, the rankings may be misguiding in the long run, unless reviews are done so frequently, which is unlikely. This is because health systems are highly evolving organizations. Given that the rankings are done at one point in time, the ranking of a health facility may not reflect its true position within the next few months following the ranking exercise. So, then if patients consider rankings 
that were done say a year ago to select their health service provider, it is likely that they would be making a choice of a provider that wouldn't quite be considered the best at that point in time.

Among the criteria used is level of professional development. This element, however, may not provide patients with all the information that they need. For instance, hospitals may list their range of specialists, say cardiologists, nephrologists and gynecologists among others. In the event the specialties considered in the ranking do not cover some rare ailments, such patients may not know where to seek help. They may also not know which hospital best handles their disease except if performance in one considered specialty predicts performance in their case which is not covered within the ratings.

Some of the aspects used such as mortality reports may not give credible comparisons. This is because reports are obtained from various facilities at different times or even days. Therefore, ranking based on this element may be considered invalid since hospitals evaluated earlier may appear to have lower mortality rates when that is not really the case. Evaluation may be unreliable too considering the fact that the hospitals in question are being rated without due regard to their initial capital base, accessible resources as well as aspects such as donor funding among other considerations which may give other facilities an upper hand over the others.

\section{Conclusion}

Healthcare ranking systems have been implemented in many parts of the world. Though they have had a few shortcomings, they generally have improved the quality of healthcare provision. They have most importantly influenced better choices by patients concerning their healthcare providers. This framework has proven effective and if applied to the developing countries; depending on respective country resource endowment among other factors would see the general world health care system transformed to a different level.

\section{References}

1. Zuger A (2015) Hospital ratings: a guide for the perplexed. JAMA 313: 1911-1912. [Crossref]

2. Harder B, Comarow A (2015) Hospital quality reporting by US News \& World Report: why, how, and what's ahead. JAMA 313: 1903-1904. [Crossref]

3. Rothberg MB, Morsi E, Benjamin EM, Pekow PS, Lindenauer PK (2008) Choosing the best hospital: the limitations of public quality reporting. Health Aff (Millwood) 27: 1680-1687. [Crossref]

4. Austin JM, Jha AK, Romano PS, Singer SJ, Vogus TJ, et al. (2015) National Hospital Rating Systems Share Few Common Scores and May Genertae Confusion Instead of Clarity. Health affairs 34: 423-430.

5. McCarthy N (2015) US Hospital Rating Systems Rarely Agree, Study Finds. BMJ British Medical Journal (Online) p. 350.

6. Sneh K (2015) Healthcare in the Middle East: An Opportunity to Innovate. Aetna News \& Analysis.

7. Jamal H, Virasakdi C, Kai HP, Nicola P, Yap MT, et al. (2012). Health and Healthcare Systems in Southeast Asia.

8. Britnell M (2015) In search of the Perfect Health System. London: Palgrave p. 59.

9. Ramya K (2013) More People Opting for Private Healthcare. Chennai, India: The Hindu.

10. Chongsuvivatwong V, Phua KH, Yap MT, Pocock NS, Hashim JH, et al. (2011) Health and health-care systems in southeast Asia: diversity and transitions. Lancet 377: 429437. [Crossref]

Copyright: (C2017 Engineer C. This is an open-access article distributed under the terms of the Creative Commons Attribution License, which permits unrestricted use, distribution, and reproduction in any medium, provided the original author and source are credited. 